\title{
Energy Efficient Decision based Routing Technique using Fog Computing Paradigm
}

\author{
Loveleen Kaur, Rajbir Kaur, Navroop Kaur
}

\begin{abstract}
In the era of new technologies, Fog computing becomes very popular in today's scenario. Fog computing paradigm brings a concept that extends cloud computing to the edge and close proximity to the Internet of Things (IoT) network. The fundamental components of fog computing are fog nodes. Additionally, fog nodes are energy efficient nodes. Numerous fog nodes are deployed in the associated fields that will handle the Internet of Things (IoT) sensors computation. Meanwhile, the Internet of Things (IoT) faces challenges, among which energy efficiency is one of the most prominent or critical challenges in the current scenario. However, sensor devices are an energy constraint that creates hotspot during the routing process. For this reason, to handle such constraints, this paper presents an effective hotspot mechanism using fog nodes that demonstrate the routing process and directed the sensors to choose the routing path as selected by the fog node. Moreover, fog node will act as a decision maker node and maintain the energy efficiency of sensors during the routing as fog nodes are energy efficient nodes. As it moves towards the emergency situation, the most appropriate and effective routing approach has been designed who maintain the energy level of sensors will be high during the routing process. The proposed routing technique could be better performance for the sake of efficient routing in terms of energy consumption and prolonging network lifetime.
\end{abstract}

Index Terms: Fog Computing, IoT, Energy Consumption, Fog nodes and Hotspot.

\section{INTRODUCTION}

Internet of Things (IoT) is a rigorous growth of the today's present Internet, which has been changed from giving human interconnection into a network of interconnected devices [1]. Internet of Things (IoT) is a continuously developing system that consistently interconnects an enormous number of heterogeneous, smart devices (things) with the Internet. With this, the association does not expect human-should human or human-to-PC cooperation. IoT was initially presented to the community in 1999 for supply chain management[2], and afterward the idea of "seeming well and good data without the help of human intercession" was broadly adjusted to different fields for example, home, transports, healthcare, environments and agriculture [3], [4]. IoT acquires novel computing and technologies and communication architectures [5]. IoT frameworks can depend on Wireless

Revised Manuscript Received on June 15, 2019.

Loveleen Kaur, Computer Science and Engineering, Punjabi University, Patiala, India.

Dr.Rajbir Kaur, Electronics and Communication Engineering, Punjabi University, Patiala, India.

Dr.Navroop Kaur, Computer Science and Engineering, Guru Nanak Dev University, Amritsar, India.
Sensor Networks (WSNs) for information gathering and their computational abilities might be improved by utilizing cloud and fog/edge computing [6], [7]. Thus, numerous applications and services of IoT have been made; including environmental and health care monitoring, smart homes, smart cities, smart grids, smart energy and smart agriculture [8]. IoT means to make the Internet pervasive and universal, and has the ability to influence numerous parts of clients' quality of life. In IoT structure, the connected organized heterogeneous devices are normally furnished with sensors, actuators, wireless transceivers, controlling processors, and an energy source (e.g., a battery) to observe their environment and also, send or receive the information. Envisioned applications for IoT traverse an extensive variety of fields and one of the predominant hindrances to actualizing such a self important plan is providing sufficient energy to work the network in an independent way without trading off the quality of service (QoS). In this manner, it is essential to overhaul the energy efficiency and effectiveness of various devices in IoT [9].

\section{A. Energy Efficiency in Internet of Things (IoT)}

Energy efficiency is an elemental factor of IoT devices. The principle goal of internet of Things (IoT) is to build the relationship of hardware objects with the physical world furthermore, to change over the information accumulated by these objects into accommodating data with no human guide. The hardware objects are constituted with battery-powered embedded sensors, communication systems, and actuators [10]. The restricted battery energy of hardware components is expended while gathering and transmitting information. The accuracy of the extracted information is higher when more of the data are gathered and collected. But this will cost more energy to be consumed. In view of energy constraints, there is an essential need to keep up a tradeoff between energy consumption by IoT systems and nature of the data extracted. In addition, the IoT resources lifetime relies on availability of the energy. The entire system under observation is affected by loss of energy. Consequently, there is an imperative need to minimize energy utilization for expanding the resources lifetime and improving operation of the IoT systems. 


\section{B. Energy Consumption in Routing}

The Consumption of Energy during routing remains a challenge in smart environments because of the restricted battery limit of sensors. Sensors are deployed in the broad range of geographical area through some wireless communication technology. The whole network can get paralyzed, if due to any reasons sensor nodes fail in these areas. Due to some internal reasons or some environmental issues like external physical damage etc, sensor nodes may stop working. Sensor devices by and large run [11] on batteries that are generally difficult to empower once installed, energy efficiency is a fundamental issue for extending the lifetime of network. Sensor based devices will work efficiently if the battery level of sensors is high. They will fail to operate in case of low battery level. Sensors like wearable devices, BP meter, and Biochip transponders on farm animals, vehicles with inherent sensors, Heart monitoring implants and DNA investigation gadgets and so on, gather valuable information with the assistance of different existing advances and after that independently stream the information between different gadgets. These sensor devices are required to be periodically recharged. As the sensors are in working environment, their battery level will get low with time. For recharging, they need to be plugged in with the electrical supply. To deal with such critical conditions, some mechanism for the sensors while considering the energy efficiency of the IoT system will be essentially required.

\section{Hotspot Problem}

In a typical smart IoT environment, sensors transmit the data with the use of actuators from one sensor to another using fixed static routing. Sensors being inherently battery based systems will fail to operate in case of low battery level. Sensors are not capable to take decision to choose the shortest and efficient path in case of occurrence of hotspot in the routing process. In wireless sensor networks-the hotspot problem means that the sensor nodes closest to the central node are basic for the lifetime of the sensor arrange in light of the fact that these nodes are required to send large number of data than nodes which are further far from the central node [12]. Also, Hotspot deals with the term where one or more sensors face the problem of high heating because most of the data is passing through these associated sensors. If the sensors are provided with the capability to take decisions, they will consume higher energy or battery. Hence sensors cannot be made decision making devices. With the use of fixed static routing, in case of emergency of hotspot occurrence in the system the whole network can shutdown. In order to deal with such type of critical conditions, there must be some mechanism or any another efficient node who will take decision in an efficient manner. Hence, Fog node is used to take decision in such type of critical conditions as fog nodes themselves are energy efficient nodes.

\section{Fog Computing}

Fog word comes from the concept of Fog computing. Fog computing is the term actually made by Cisco to compute and describe various supporting applications of IoT [13]. Fog computing is the extension of cloud computing or simply fog is a cloud near the ground and enables a new breed of services and applications. The idea of fog computing is proposed to provide cloud like services to the nearby edge devices and also help the conventional cloud computing [14]. In an environment, IoT systems produce unprecedented measures of information that could be of use in lots of ways. The wait brought on by moving information to the cloud for processing and back again to the application is not acceptable in the various latency-based applications [15]. Such a great amount of information send to the cloud for processing, storage and handling, since it would not flexible and soak the network transfer speed. Moreover, Fog computing is just a dispersed computing criterion that gives cloud-like administrations or services close by the edge devices or gadgets. It supports cloud and an edge resource alongside utilizing it does possess foundation. Fundamentally, the technology handles Internet of Things (IoT) information locally by utilizing customers or edge gadgets close clients to transport out a significant limit of capacity, control, communication, setup, and administration. The methodology profits by edge gadgets' closeness to sensors nodes, while using the on-demand adaptability of cloud resources. Fog systems generally use the sense-process-actuate and stream-processing programming models. Sensors stream information to IoT systems, applications running on fog nodes buy in to and process the data, and the acquired bits of knowledge are made an interpretation of into activities sent to actuators. Fog nodes are the fundamental components of fog computing that can be structured and installed [16] in various approaches to best fit the requirements of the various applications of IoT that keep running on them as shown in figure.1.

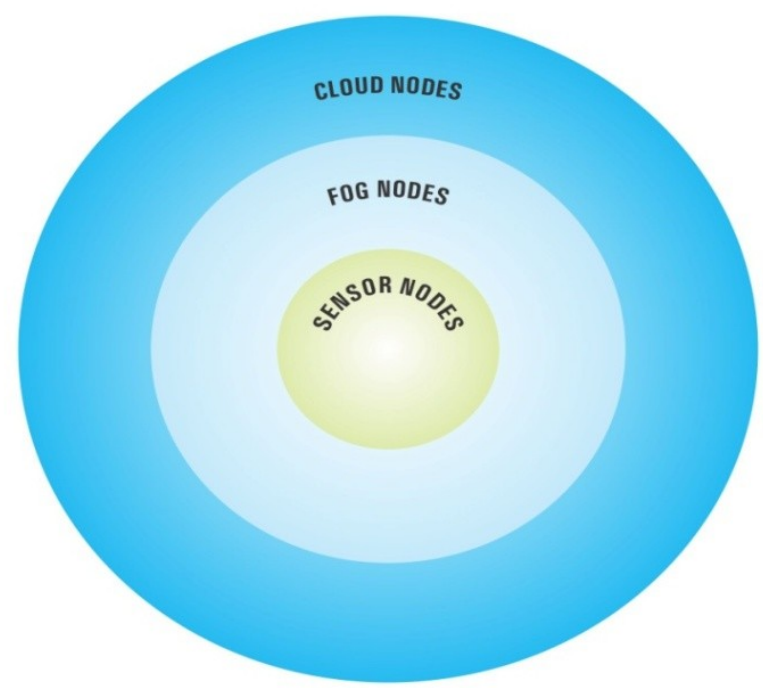

Fig. 1 Fog Computing Environment 
The physical and topographical situating of fog nodes are set in pecking order, topology, the numbers, protocols, types, and information data transfer capacity limits of the connections between fog nodes, things, and the cloud, the software and hardware plan of individual fog nodes, and how an entire IoT system is coordinated and overseen [17]. Fog nodes include edge routers, access points, and switches, set top boxes, end-user devices and Base Stations etc. In addition, fog nodes can likewise be associated with cloud by Internet so as to utilize the rich computing and cloud storage assets [18]. Fog nodes are processed and stored the data produced by sensors and edge devices. At that point, the remaining valuable data is transferred for storage or next 80 processing to the cloud server. Fog computing serve as greener computing through the joint effort with the conventional cloud computing model and also help cloud computing to play its esteem all the more proficiently [19] [20].

\section{E. Fog Computing Characteristics}

The structure parameters of both the Cloud and the Fog are computation, storage, and network resources. However, a number of characteristics that describes the role of Fog computing.

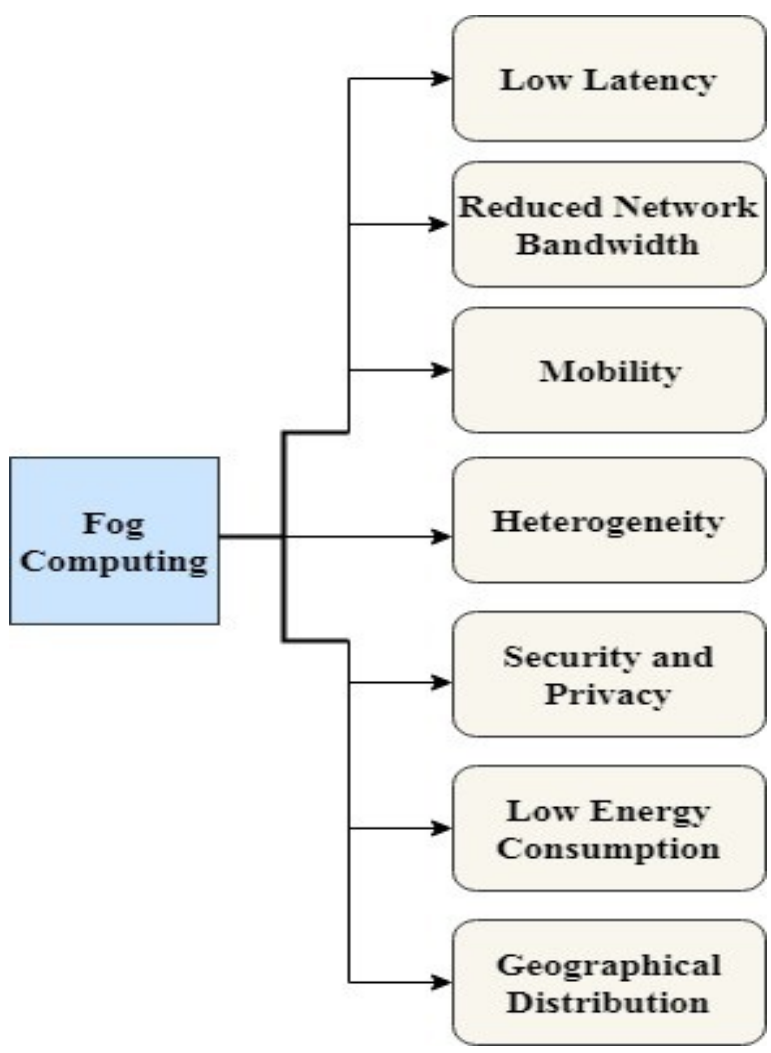

Fig. 2 Fog Computing Characteristics

\section{F. Low Energy Consumption Fog Nodes}

In Fog computing environment, Fog nodes are geographically distributed. In this way, it won't produce a large amount of heat because of fixation, and there is no need of additional cooling framework. Furthermore, Fog nodes help to reduce power consumption, diminishing cost, and also saving energy in fog computing paradigm. Fog computing gives greener computing standard. Moreover, as it is worked out, the energy utilization of the worldwide Internet of Things (IoT) will turn into an extreme issue. Likewise, numerous things or items, including sensors, advanced mobile phones, etc, will have energy limitations forced by their size, weight, and internal battery limit. Fog methods can help with such these limitations. Smart agriculture is one of the illustration use case with significant energy requirements. In farm, it may have several sensors dispersed all through its fields estimating soil moisture, crop health and developing conditions and looking for indications of pests. Furthermore, sensors are frequently battery power-driven and truly energy obliged in IoT. In this scenario, sensors periodically awake, get a few readings, send the crude information to the fog utilizing a low-control radio connection and backpedal to low-power rest mode. Fog permits the sensor system to utilize to great degree low-power methods, and deals with the energy-escalated calculation and WAN capacities for their benefit.

The rest of the paper is organized as follows. Section 2 presents related work of existing routing techniques and frameworks. Proposed approach design is displayed in Section 3. Section 4 presents results and discussion of proposed work. Section 5 concludes the paper and discusses some future work.

\section{RELATED WORK}

Some of the issues have been observed in the existing works of Sensors Routing in IoT. In paper [21] proposed an energy efficient technique for IoT applications which is based on three variables to find the best next hop in routing process. Provides the platform [22] to improve the energy efficiency of various smart gadgets and also introduces the genetic algorithm for clustering. A simulation result shows on the basis of energy consumption, end-to-end delay, bit error rate, and throughput. In [23], proposed an energy harvesting sensor networks novel routing algorithm that works to maintain the energy consumption, network lifetime and also introduce an energy harvesting sensor networks outline on methodology of an optimal power control to optimize the outage probability for bidirectional sub-channels in this scheme. In [10], proposed an energy efficient architecture of IoT which consists of three layers that are used to enhance the energy efficiency of sensor nodes based on some parameters and also enhanced asset usage of cloud assets. In paper [24] introduced the framework that is also based on three layers that is very suitable for the utilization of IoT resources and the energy consumption. Paper [25] ensures to save the sensor nodes to make sure minimal power cost and comparatively low residual

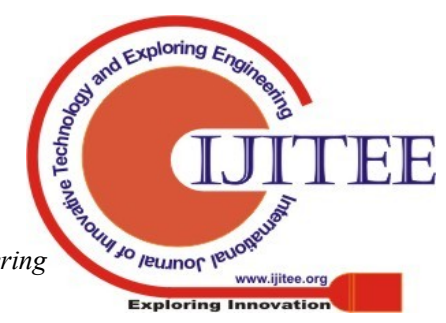


energy during this data relay process. It emphasizes on maximal network life and minimal energy consumption in this process. Paper [26] provides a method called Delay Iterative Method (DIM) that is used for estimating delay and also provide the solution to take no notice of reliable paths in this system. In paper [27] a cluster based aware energy routing that is used to expand the system throughput by diminishing the end-to-end delay, less packet drop proportion and enhancing system lifetime. In [28] SGEAR can provide stable routing decisions for sensor nodes and the energy of the system can at present consume uniformly and also this is more appropriate and draws out the lifetime of Wireless Sensor Networks when it is joined with sleeping scheduling scheme in this algorithm. In this paper [29], a scheme has been proposed that is used to stable the network load by concentrating on node residual energy and target is to sort out maximal system use, while keeping end-to-end delay underneath an endorsed limit likewise, it estimates the single-hop postponement at relay node for every transmission and also enables the relay nodes to utilize as it were the adjacent or neighborhood channel data and evaluated postponements to route the packets in this scheme.

Researchers have been focused on various parameters of sensor routing in existing work. Moreover, as per the existing works, research effort has been implemented and analyzed through sensors routing and solving the problems in terms of network lifetime, bit rate ratio, end-to-end delay and throughput to save the network energy of nodes. None of the researchers have been focused on dynamic sensor routing with energy efficient mechanism using fog computing. Fog computing can be employed to optimize network bandwidth and also maintain the energy efficiency of fog nodes. For this reason, this paper focuses on the dynamic hotspot mechanism for sensor routing based on fog nodes. However, Fog nodes will help to manage the sensor routing with an energy efficient manner as fog nodes are energy efficient nodes.

\section{PROPOSED WORK}

The conventional routing techniques are not prepared to fulfill the necessities of the present situation as they lack in fundamental requirements like energy consumption, network cost, etc. To clarify the issue of conventional routing techniques, there is a need to develop an energy efficient technique. Moreover, for the development of energy efficient technique, the following points should be considered for the progress of sensor routing:-

1. The energy consumption of sensors will be high when the sensors' capacity to take decisions increases. With the use of more energy, the battery level will get low with time. This can create network performance-related problems in the system or shut down the entire system. In this manner, it is essential to make suitable tradeoff between least energy utilization and maximum system or network lifetime. However, In order to deal with this critical condition, there must be some corrective mechanism in the network or an alternate node that will take decisions in an efficient manner in the sensors' routing process. Fog node is an energy efficient node that can take decisions in such a type of critical condition.

2. Several Energy Efficient routing algorithms are available but the limitation of most of these is that fixed static routing is used and in case of any hotspot occurrence between network sensors, the entire network might shut down. As the sensor devices have energy constraints and researchers have always been wanting to maintain the sensors' energy level for longer periods of time. In existing works sensors' routing is fixed. To make the sensors' routing more effective and energy efficient, a dynamic decision - making node is needed, which will direct how a sensor will route to the next sensor in the routing process. If any hotspots between sensors occur due to some internal or external environment issues then the routing process will not be possible. The possible solution of the hotspot problem is to place additional energy efficient nodes which will take the decisions to choose other shortest paths between the sensors nodes. This is where our work comes into play and proposed energy efficient routing of sensors is the main difference from the previous works.

In this proposed work, we focused on the most effective and energy efficient routing technique. In this technique, to achieve our goal, an Energy-Efficient Fog Node will act as a decision- maker node which will efficiently decide to route in which direction the sensors will route in the network. The sensors will dynamically communicate with fog nodes which will direct the sensors to route on which best direction choosing the shortest path. Subsequently, the sensors will route according to the paths selected by the Fog nodes as shown in figure.3.
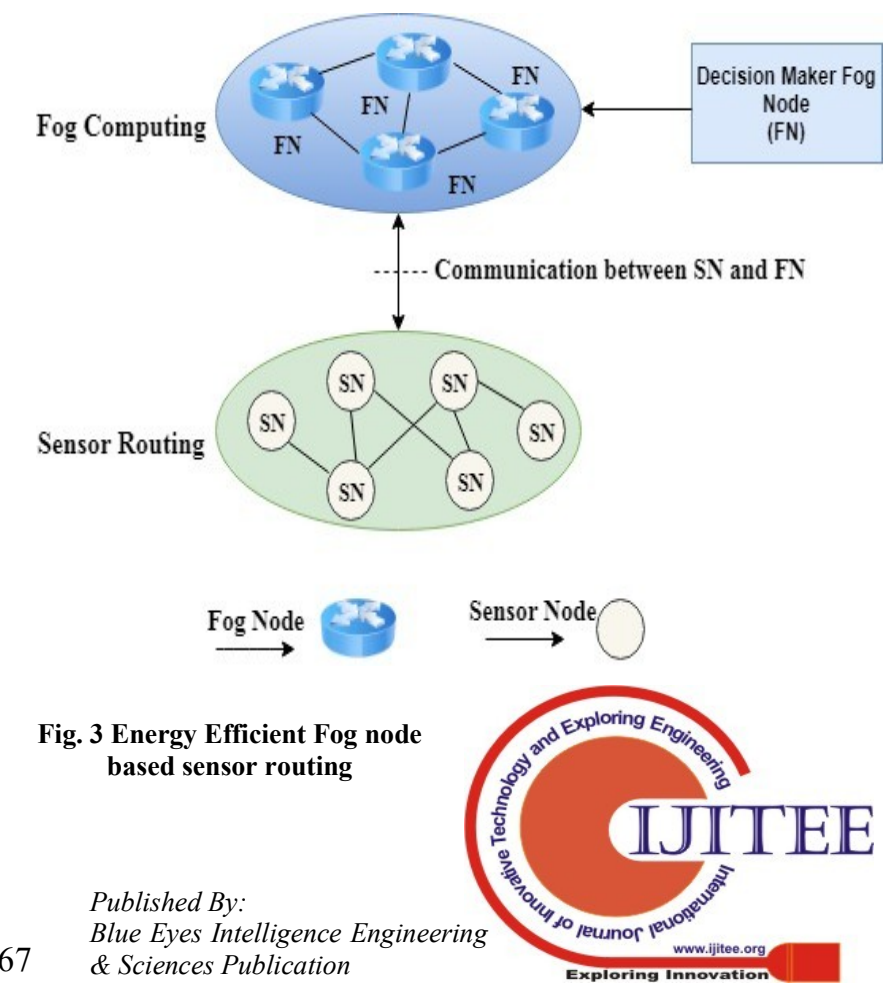
By using Fog node some delay will be created in case of emergency conditions or situations while the network will continue to work efficiently in absence of emergency conditions. However, emergency situations need negligible latency. To achieve this proposal, the system is designed in such a way so as to work according to the current status of the system as shown in figure. 4 .

a. If Emergency occurs, then the system will go through the fixed shortest route i.e. it will work according to default operation.

b. But in case of non-emergency situation, the system will work according to the dynamic shortest route with an energy efficient fog node.

At time $\mathrm{T}$,

$$
\begin{gathered}
\text { Allocate Fog Node FN } \\
\text { When FN triggers, } \\
\text { Emergency then, }
\end{gathered}
$$

Default operation.

Otherwise

Dynamic shortest route using fog node.

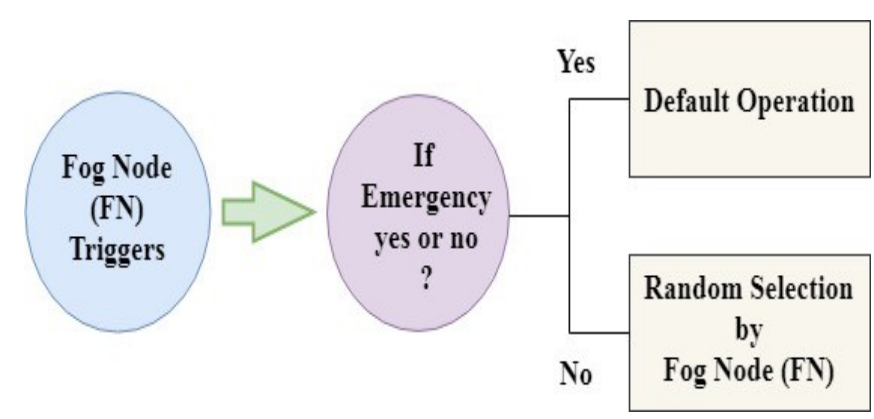

Fig. 4 Fog node based Emergency-Situation routing

The algorithm of proposed technique is as follows:

1. Start.

2. Set Fog nodes as $\mathrm{FN}_{\mathrm{s}}$ and Sensor nodes as $\mathrm{SN}_{\mathrm{s}}$.

3. Configure all $\mathrm{FN}_{\mathrm{s}}$ and $\mathrm{SN}_{\mathrm{s}}$ with their bandwidth and processing time.

4. A tree has been formulated with

a. Fog nodes as Parent.

b. Set all IoT sensor nodes at terminal node.

5. Make a connection between $\mathrm{FN}_{\mathrm{s}}$ and $\mathrm{SN}_{\mathrm{s}}$.

6. At time $\mathrm{T}$, when $\mathrm{FN}$ triggers,
- If emergency-yes,

SN sends data to next corresponding sensor in routing i.e. default operation.

- If emergency-no,

FN decision and random selection dynamic method.

7. Energy consumption is calculated based on various parameters of battery operated sensor nodes.

8. Network cost of complete system is calculated and recorded in different scenarios.

9. The comparative graphs have been plotted.

10. End.

\section{EXPECTED OUTCOME AND DISCUSSION}

The proposed work demonstrates the energy efficient technique that will handle routing in a very efficient manner. The outcomes of this technique will be evaluated through various parameters. Based on these parameters fog nodes will choose an efficient path and direct the sensors to route on the best direction that will be energy efficient. In this whole scenario, when it moves an emergency situation the fog node will trigger to check whether the emergency or not. If an emergency occurs then the sensor nodes will work accordingly set as previously existing techniques i.e. sensor node to sensor node data communication. But in case of the non-emergency situation, the sensor node will communicate to the next sensor node as directed by fog node that will be energy efficient, the same as fog nodes are energy efficient nodes. However, this new dynamic method based on fog node will lead to the low energy consumption of the whole system.

\section{CONCLUSION}

This paper focuses on the concept of fog node that handles the sensor routing to improve the energy efficiency of the IoT sensors nodes which are deployed in any environment. This method improves the energy efficiency of various sensor nodes and also maintains the network lifetime of the existing networks. This paper focuses on the routing process of sensors deployed in different regions. The processing of routing is handled by fog node as acting as a decision maker node. Fog node will direct the sensors to route on which direction in routing. Moreover, this will head to lead the avoidance of hotspot occurrence and maintain the energy efficiency of the network.

\section{REFERENCES}

1. O. Bello and S. Zeadally, "Intelligent Device-to-Device Communication in the Internet of Things," Ieeexplore.Ieee.Org, vol. 10, no. 3, pp. 1-11, 2015 .

2. Kevin, "That 'internet of things' thing," RFID J., vol. 22, no. 7, pp 97-114, 2009

3. K. Gusmeroli, S., Haller, S., Harrison, M., Kalaboukas, K., Tomasella, M., Vermesan, O., \& Wouters, Vision and challenges for realizing the internet of things, vol. 1, no. APRIL. 2009.

4. J. Gubbi, R. Buyya, S. Marusic, and M. Palaniswami, "Internet of Things (IoT): A vision, 
architectural elements, and future directions," Futur. Gener. Comput. Syst., vol. 29, no. 7, pp. 1645-1660, 2013.

5. S. Engineering, M. Program, G. Square, and E. Zayed, "An Energy-Efficient Multi-Objective Scheduling Model for Monitoring in Internet of Things," vol. 4662, no. c, pp. 1-12, 2012.

6. W. Shi, J. Cao, Q. Zhang, Y. Li, and L. Xu, "Edge Computing: Vision and Challenges," IEEE Internet Things J., vol. 3, no. 5, pp. 637-646, 2016.

7. J. Lin, W. Yu, N. Zhang, X. Yang, H. Zhang, and W. Zhao, "A Survey on Internet of Things: Architecture, Enabling Technologies, Security and Privacy, and Applications," IEEE Internet Things $J$., vol. 4, no. 5, pp. $1125-1142,2017$.

8. S. He, J. Chen, and Y. Sun, "Coverage and connectivity in duty-cycled wireless sensor networks for event monitoring," IEEE Trans. Parallel Distrib. Syst., vol. 13, no. 3, pp. 475-482, 2012.

9. P. Kamalinejad, C. Mahapatra, Z. Sheng, S. Mirabbasi, V. C. M. Leung, and Y. L. Guan, "Wireless energy harvesting for the Internet of Things," IEEE Commun. Mag., vol. 53, no. 6, pp. 102-108, 2015.

10. N. Kaur and S. K. Sood, "An Energy-Efficient Architecture for the Internet of Things (IoT)," IEEE Syst. J., pp. 1-10, 2015.

11. M. Dong, K. Ota, and A. Liu, "RMER: Reliable and Energy-Efficient Data Collection for Large-Scale Wireless Sensor Networks," IEEE Internet Things J., vol. 3, no. 4, pp. 511-519, 2016.

12. Y. Bi, L. Sun, J. Ma, N. Li, I. A. Khan, and C. Chen, "HUMS: An autonomous moving strategy for mobile sinks in data-gathering sensor networks," Eurasip J. Wirel. Commun. Netw., vol. 2007, 2007.

13. R. Mahmud, R. Kotagiri, and R. Buyya, "Fog Computing: A Taxonomy, Survey and Future Directions," pp. 1-28, 2016.

14. F. Bonomi, R. Milito, J. Zhu, and S. Addepalli, "Fog Computing and Its Role in the Internet of Things," Proc. first Ed. MCC Work. Mob. cloud Comput., pp. 13-16, 2012.

15. A. Dastjerdi, R. B.- Computer, and undefined 2016, "Fog computing: Helping the Internet of Things realize its potential," Ieeexplore.Ieee.Org, 2016.

16. C. C. Byers, "Architectural Imperatives for Fog Computing: Use Cases, Requirements, and Architectural Techniques for Fog-Enabled IoT Networks," IEEE Commun. Mag., vol. 55, no. 8, pp. 14-20, 2017.

17. $\mathrm{P}$. Hu, S. Dhelim, H. Ning, and T. Qiu, "Survey on fog computing: architecture, key technologies, applications and open issues," J. Netw. Comput. Appl., vol. 98, pp. 27-42, 2017.

18. M. Aazam and E. N. Huh, "Fog Computing: The Cloud-IoT/IoE Middleware Paradigm," IEEE Potentials, vol. 35, no. 3, pp. 40-44, 2016.

19. M. Hajibaba and S. Gorgin, "A review on modern distributed computing paradigms: Cloud computing, jungle computing and fog computing," $J$. Comput. Inf. Technol., vol. 22, no. 2, pp. 69-84, 2014.

20. M. Yannuzzi and R. Milito, "Key ingredients in an IoT recipe Fog Computing,.pdf," pp. 325-329.

21. M. Elappila, S. Chinara, and D. R. Parhi, "Survivable Path Routing in WSN for IoT applications," Pervasive Mob. Comput., vol. 43, pp. 49-63, 2018

22. F. Zawaideh, "An Energy Efficient Clustering Algorithm for Wireless Sensor Networks ( EECA )," no. September, pp. 39-44, 2012.

23. F. Li, M. Xiong, L. Wang, H. Peng, J. Hua, and X. Liu, "A novel energy-balanced routing algorithm in energy harvesting sensor networks," Phys. Commun., 2018.

24. K. Wang, Y. Wang, Y. Sun, S. Guo, and J. Wu, "Green Industrial Internet of Things Architecture: An Energy-Efficient Perspective," IEEE Commun. Mag., vol. 54, no. 11, pp. 48-54, 2016.

25. J. Luo, J. Hu, D. Wu, and R. Li, "Opportunistic routing algorithm for relay node selection in wireless sensor networks," IEEE Trans. Ind. Informatics, vol. 11, no. 1, pp. 112-121, 2015.

26. T. Qiu, Y. Lv, F. Xia, N. Chen, J. Wan, and A. Tolba, "ERGID: An efficient routing protocol for emergency response Internet of Things," $J$. Netw. Comput. Appl., vol. 72, pp. 104-112, 2016.

27. S. B. Shah, Z. Chen, F. Yin, I. U. Khan, and N. Ahmad, "Energy and interoperable aware routing for throughput optimization in clustered IoT-wireless sensor net," Futur. Gener. Comput. Syst., vol. 81, pp. 372-381, 2018.

28. D. Sun, X. Huang, Y. Liu, and H. Zhong, "Predictable Energy Aware Routing based on Dynamic Game Theory in Wireless Sensor Networks," Comput. Electr. Eng., vol. 39, no. 6, pp. 1601-1608, 2013.

29. S. Wen, C. Huang, X. Chen, J. Ma, N. Xiong, and Z. Li, "Energy-efficient and delay-aware distributed routing with cooperative transmission for Internet of Things," J. Parallel Distrib. Comput., 2017.

\section{AUTHORS PROFILE}

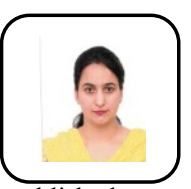

Loveleen Kaur is an Assistant Professor in Khalsa College of Engineering and Technology, Amritsar. She has completed her M.Tech. from Guru Nanak Dev Engineering College, Ludhiana and currently doing Ph.D from Punjabi University Patiala, Punjab, India. She has published more than 10 papers in International Journals. And more than 5 papers in National / International Conferences. Her areas of research are Big Data, IoT, Cloud computing, Fog computing and Edge computing.

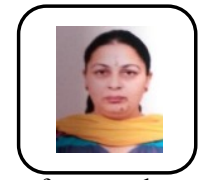

Dr. Rajbir Kaur is an Assistant Professor in Punjabi University Patiala and completed her Ph.D from Punjabi University Patiala, Punjab, India. She has published more than 30 papers in International Journals. And more than 10 papers in National / International Conferences. Her areas of research are Analog and Digital communication, Networking, Fog computing and Signal Processing.

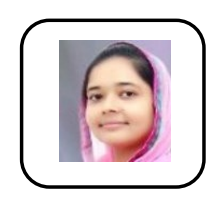

Dr. Navroop Kaur has done Ph.D from Guru Nanak Dev University Amritsar, Punjab, India. She has published more than 20 papers in International Journals. And more than 10 papers in National / International Conferences. Her areas of research are Cloud Computing, Data Mining, Internet of Things, Big Data, Performance Evaluation, Game Theory, Fog computing. 